\title{
Examining the Effects of Common Laboratory Methods on the Sensitivity of Carbon Fiber Electrodes in Amperometric Recordings of Dopamine
}

\author{
William T. Prater, Malli Swamy, Megan D. Beane, Deranda B. Lester* \\ Department of Psychology, The University of Memphis, Memphis, TN, USA \\ Email: wtprater@memphis.edu, swamymalli1@gmail.com, mbeane@memphis.edu, ^dbrewer@memphis.edu
}

How to cite this paper: Prater, W.T., Swamy, M., Beane, M.D. and Lester, D.B. (2018) Examining the Effects of Common Laboratory Methods on the Sensitivity of Carbon Fiber Electrodes in Amperometric Recordings of Dopamine. Journal of Behavioral and Brain Science, 8, 117-125. https://doi.org/10.4236/jbbs.2018.83007

Received: January 9, 2018

Accepted: March 3, 2018

Published: March 7, 2018

Copyright $\odot 2018$ by authors and Scientific Research Publishing Inc. This work is licensed under the Creative Commons Attribution International License (CC BY 4.0).

http://creativecommons.org/licenses/by/4.0/

\begin{abstract}
Carbon fiber microelectrodes (CFEs) are useful when combined with electrochemical techniques for measuring changes in neurotransmitter concentrations. We addressed conflicting details regarding the use of CFEs. Experimental groups consisted of CFEs at different ages (1 week, 1 month, or 2 months), cleaned in solvents (isopropanol or xylene), and exposed to in vitro use (flow cell calibrations) or in vivo use (in brain tissue). In order to determine if any of these factors affect CFE sensitivity, the present study utilized fixed potential amperometry and a flow injection system to calibrate CFEs for the measurement of dopamine. The sensitivity index $(\mathrm{nA} / \mu \mathrm{M}$ per $100 \mu \mathrm{m}$ of exposed carbon fiber) was not affected by the age of CFEs or pre-cleaning with xylene or isopropanol. CFE sensitivity of the in vitro exposure group also did not differ from untreated CFEs, indicating the calibration process did not alter sensitivity. However, in vivo use in brain tissue did reduce sensitivity. This effect was negated and sensitivity restored by cleaning CFEs in isopropanol or xylene following in vivo brain recordings. Given that variations in CFE sensitivity can skew results, our findings can help standardize CFE use and explain discrepancies between researchers.
\end{abstract}

\section{Keywords}

Fixed Potential Amperometry, Carbon Fiber Electrode, Dopamine, Flow Injection System, Electrode Calibration

\section{Introduction}

Carbon fiber electrodes (CFEs) have been in use for neuroscience research since 
the late 1970s when Pujol and colleagues introduced them in combination with normal pulse polarography and differential pulse voltammetry to measure the oxidation of several neurotransmitters, including dopamine, norepinephrine, and serotonin [1] [2]. Carbon fibers provide several benefits including relatively inert electrochemistry, electrocatalytic activity for a variety of redox reactions, and low cost [3]. Furthermore, importantly for neuroscience research, carbon fibers are biologically well-suited in that they are non-toxic to neurons and can be less than $10 \mu \mathrm{m}$ in diameter so as to not damage brain tissue upon implantation [4]. Currently, the most common techniques utilizing CFEs for the measurement of neurotransmitter release include fixed potential amperometry (sometimes called continuous amperometry) [5] [6] [7] and fast scan cyclic voltammetry (FSCV) [8] [9]. Fixed potential amperometry involves applying a fixed continuous potential to the CFE allowing for quantification of analytes that oxidize below the set potential. FSCV involves applying a potential in a series of triangular-shaped waveforms, for example cycling from $-0.4 \mathrm{mV}$ to $1.2 \mathrm{mV}$ and back to $-0.4 \mathrm{mV}$, allowing for exact measurement of the analyte's oxidation and reduction, consequently providing a chemical selectivity lacking in fixed potential amperometry. However, fixed potential amperometry boasts the greatest temporal resolution, measuring up to 10,000 samples per second compared to generally 10 samples per second using FSCV. Thus, each electrochemical technique has advantages and disadvantages, but both are especially well known as being effective and reliable for quantifying phasic dopamine release using CFEs [10].

Although CFEs are available for purchase (see World Precision Instruments in Sarasota, FL, Kation Scientific in Minneapolis, MN, and Pinnacle Technology in Lawrence, KS), most labs construct CFEs in house. Generally, the most common method for CFE construction involves threading a carbon fiber through a glass tube and heat-pulling the glass to form a seal around the carbon fiber. A stainless steel wire is inserted into the tube, and graphite powder is fed into the tip to ensure contact between the fiber and the wire. The carbon fibers are then cut to the desired length (generally $100-1000 \mu \mathrm{m}$ ) extending from the glass seal [1] [6] [11]. These steps are relatively standard and have been since Ponchon et al. (1979) first published their methods for constructing CFEs [2].

The present study aimed to address less consistent and/or rarely described details regarding the use of CFEs and to determine whether such details influence the sensitivity of CFEs. For example, researchers rarely discuss the lag time between CFE construction and use (i.e., the age of the CFE). Some researchers clean CFEs prior to use with solvents such as isopropanol [9] [12] or xylene [13], while others report no cleaning procedures [6] [7]. Furthermore, to ensure CFE responsiveness, some researchers calibrate CFEs prior to experimental use, while others prefer to calibrate following experimental use [8] [11]. Rarely is it mentioned whether cleaning takes place again between experimental use and calibrations or vice versa. In order to determine if any of these factors affect the sensi- 
tivity of CFEs, the present study utilized fixed potential amperometry and a flow injection system to calibrate CFEs for the measurement of dopamine in solution. The results of the present study should help standardize use of CFEs and may explain discrepancies between researchers given that variation in CFE sensitivity can skew results, especially if attempting to compare between subjects or claim absolute neurotransmitter concentrations.

\section{Materials and Methods}

\subsection{Electrode Construction}

Each CFE was constructed by threading a single $7 \mu \mathrm{m}$ o.d. carbon fiber (Goodfellow Corporation: C005722) through a borosilicate glass tube (A-M Systems: 616000). All carbon fibers were from the same manufactured lot, approximately 7 - 8 months old. The glass tube was heat-pulled using Sutter Instruments model P-80 to form a sealed tip around the carbon fiber. The sealed glass tips ranged from $500-750 \mu \mathrm{m}$. Graphite powder (Sigma-Aldrich: 282863) was packed into the tip of the pulled glass, and a metal wire inserted to make contact with the fiber. The metal wire, which is ultimately connected to the electrometer, is secured in place with glue at the glass tube end opposite the carbon fiber. The protruding carbon fiber was cut under a stereomicroscope to extend $500 \mu \mathrm{m}$ past the pulled glass tip. The exact length of each CFE was recorded and factored into the data anaylsis by expressing sensitivity per $100 \mu \mathrm{m}$ of carbon fiber. The steps used by the present study are relatively standard for detecting dopamine efflux with electrochemical techniques [6] [7] [14].

\subsection{Electrode Calibration}

To calibrate the CFEs using fixed potential amperometry, a flow injection system was used in conjunction with a silver/silver chloride reference electrode and a stainless steel auxiliary combination [11]. The CFE was connected to an electrometer to close the circuit. With $0.01 \mathrm{M}$ phosphate-buffered saline (PBS) flowing through the vessel at $8 \mathrm{~mL} /$ minute, serially increases in dopamine concentrations $(0.2,0.4,0.8$, and $1.2 \mu \mathrm{M})$ were given at the same rate. Amperometric readings consisted of applying a fixed positive potential of $0.8 \mathrm{~V}$ to the CFE with sample readings at $10,000 / \mathrm{sec}$ [7]. This calibration process allowed for the conversion of recorded dopamine oxidation currents $(\mathrm{nA})$ into dopamine concentrations $(\mu \mathrm{M})$.

\subsection{Experimental Groups}

In order to determine if the age of the CFEs affected their sensitivity, CFEs were calibrated 1 week, 1 month, and 2 months following construction. The effects of CFE sensitivity following varying chemical exposures were also examined. For this, the exposed part of the CFE was manually swirled in either xylene or isopropanol (10 seconds each) 30 minutes prior to calibration. Xylene and isopropanol are chemicals that have been previously used in published studies as prep- 
ping or cleaning agents for CFEs [13] [15].

To determine whether CFE sensitivity is altered by the calibration process itself, a group of electrodes was recalibrated with at least 1 hour between calibrations. This group is referred to as the "in vitro exposure group". To determine whether CFE sensitivity is altered by experimental use, a group of electrodes were calibrated following amperometric recordings in brain tissue. Briefly, these experiments included 2 - 3 hours of recording stimulation-evoked dopamine release in the brains of anesthetized mice using in vivo fixed potential amperometry [7] with each electrode being used in 1 brain only. This group of CFEs is referred to as the "in vivo exposure group". In order to determine if the cleaning agents had an effect on CFE sensitivity following use during in vivo fixed potential amperometry recordings, a portion of electrodes in this group were treated with xylene or isopropanol as described above (swirled immediately after removal from brain in either xylene or isopropanol for $10 \mathrm{sec}$, then allowed to dry for at least $30 \mathrm{~min}$ before in vitro calibration). See Table 1 for a list of experimental groups.

\subsection{Chemicals}

All chemicals, including xylene (214736) and isopropanol (I9516), were purchased from Sigma-Aldrich. Dopamine hydrochloride (H8502) was dissolved in $0.01 \mathrm{M}$ PBS. PBS solution consisted of $1.09 \mathrm{~g} \mathrm{Na}_{2} \mathrm{HPO}_{4}$ (anhydrous), $0.32 \mathrm{~g}$ $\mathrm{NaH}_{2} \mathrm{PO}_{4}$ (anhydrous), and $9 \mathrm{~g} \mathrm{NaCl}$ in $1000 \mathrm{ml}$ of $\mathrm{dH}_{2} \mathrm{O}$ with $\mathrm{pH}$ adjusted to 7.4 .

\subsection{Data Analysis}

See Figure 1(a) for example data showing current change over time at each dopamine concentration. Individual data points were collected $5 \mathrm{sec}$ after initiation of each dopamine concentration. Data points for each dopamine concentration were averaged to give a mean $\mathrm{nA} / \mu \mathrm{M}$ for each CFE. The mean $\mathrm{nA} / \mu \mathrm{M}$ was then

Table 1. Experimental groups.

\begin{tabular}{cccc}
\hline Experimental Exposure & Chemical Exposure & Age of Electrode & N \\
\hline None & None & 1 week & 39 \\
& & 1 month & 8 \\
None & Xylene & 2 months & 10 \\
& Isopropanol & 1 week & 12 \\
In vitro & None & 1 week & 16 \\
In vivo & None & 1 week & 10 \\
& Xylene & 1 week & 38 \\
& Isopropanol & 1 week & 9 \\
& - & 1 week & 10 \\
& & - & 152 \\
\hline
\end{tabular}



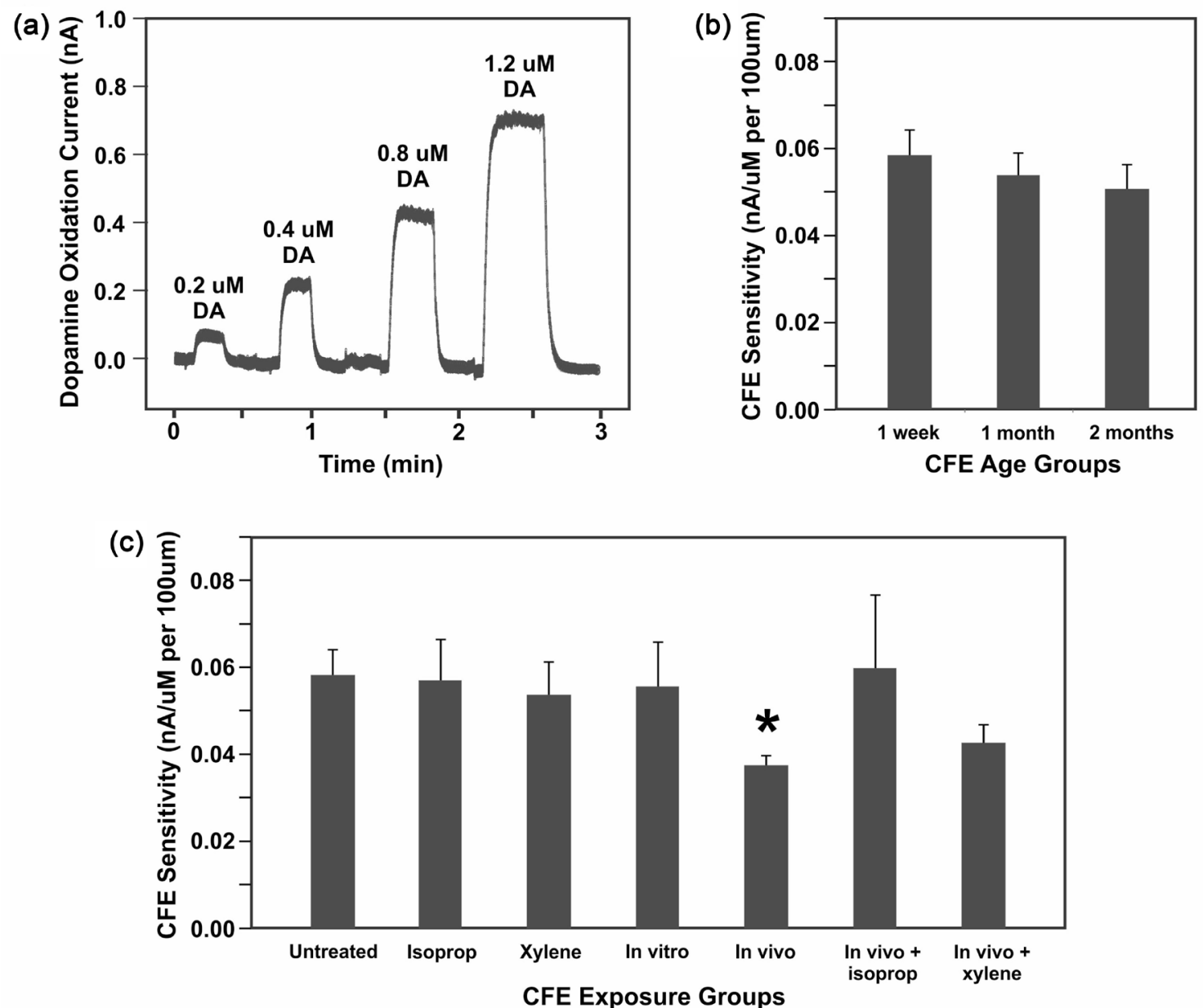

Figure 1. Amperometric recordings during in vitro calibrations of carbon fiber electrodes (CFEs) using a flow injection system. Dopamine (DA) was administered at 4 concentrations $(0.2,0.4,0.8,1.2 \mu \mathrm{M})$, as shown in this example data (a). The sensitivity of CFEs was quantified in 3 age groups (b) and 7 exposure groups (c). ${ }^{*}$ Signficantly reduced sensitivity ( $\mathrm{nA} / \mu \mathrm{M}$ per $100 \mu \mathrm{m}$ of exposed carbon fiber) of CFEs exposed to in vivo brain recordings compared to untreated CFEs.

divided by the carbon fiber length in order to generate a normalized sensitivity index corresponding to $\mathrm{nA} / \mu \mathrm{M}$ per $100 \mu \mathrm{m}$ of exposed carbon fiber. High $\mathrm{nA} / \mu \mathrm{M}$ ratio indicates high CFE sensitivity. An analysis of variance (ANOVA) was used to compare mean sensitivity indexes between ages (1 week, 1 month, or 2 months) or exposure groups (no exposure, xylene, isopropanol, in vitro, in vi$v o$, in vivo then xylene, or in vivo then isopropanol). Post-hoc tests (Fisher's LSD) were used to examine significant group differences indicated by $p<0.05$.

\section{Results}

\subsection{Effects of Age on Electrode Sensitivity}

The sensitivity index (nA/ $\mu \mathrm{M}$ per $100 \mu \mathrm{m}$ of exposed carbon fiber) was not affected by the age of CFEs. CFEs constructed 1 or 2 months prior to calibration had no significant decrease in ability to detect dopamine oxidation (mean \pm SEM: $0.537 \pm 0.006$ and $0.506 \pm 0.006$, respectively) compared to CFEs con- 
structed within 1 week of use $(0.0584 \pm 0.006)(\mathrm{F}=0.26, \mathrm{p}=0.77)($ Figure $1(\mathrm{~b}))$.

\subsection{Effects of Cleaning and Use on Electrode Sensitivity}

An ANOVA comparing the CFE sensitivity indexes of the various exposure groups (no exposure, xylene, isopropanol, in vitro, in vivo, in vivo then xylene, and in vivo then isopropanol) indicated a significant effect $(\mathrm{F}=2.18, \mathrm{p}=0.05)$. Post hoc analyses showed the sensitivity index was not affected by cleaning the CFEs with xylene or isopropanol (mean \pm SEM: $0.054 \pm 0.008$ and $0.057 \pm 0.010$, respectively) when compared to untreated (no exposure) CFEs $(0.058 \pm 0.006 ; \mathrm{p}$ $=0.64$ and 0.88 , respectively). The CFE sensitivity index of the in vitro exposure group $(0.056 \pm 0.012)$ was also not significantly different from the untreated CFEs ( $p=0.79$ ), indicating the calibration process itself does not alter CFE sensitivity. However, use in amperometric recordings in brain tissue does affect CFE sensitivity, as the sensitivity index of CFEs in the in vivo exposure group $(0.038 \pm 0.002)$ were significantly reduced compared to the untreated group $(\mathrm{p}=$ 0.003). This effect was negated by cleaning CFEs following in vivo brain recordings. The sensitivity index of the in vivo exposure CFEs cleaned with xylene $(0.043 \pm 0.004)$ or isopropanol $(0.060 \pm 0.025)$ were no different from that of untreated CFEs ( $\mathrm{p}=0.16$ and 0.88 , respectively) (Figure $1(\mathrm{c})$ ).

\section{Discussion}

CFEs have been utilized in neuroscience labs for nearly 4 decades. Several factors regarding the handling of CFEs are inconsistent and/or rarely described in publications from labs using electrochemical techniques to measure neurotransmitter efflux. Using fixed potential amperometry with CFEs to quantify dopamine (Figure 1(a)), the present study addressed some of these factors in an attempt to standardize the handling of CFEs and explain potential discrepancies in results across labs.

The present results indicate that the CFE age, at least up to 2 months, does not affect CFE sensitivity for dopamine measurement. The sensitivity indexes $(\mathrm{nA} / \mu \mathrm{M}$ per $100 \mu \mathrm{m}$ of exposed carbon fiber) generated from in vitro calibrations of 1 or 2 month old CFEs were not significantly different from those of 1 week old CFEs (Figure 1(b)). Although researchers rarely publish details in their methods regarding the time laps between CFE construction and use, anecdotally we have heard of researchers discarding CFEs after a certain period of time due to fear of reduced performance quality. To our knowledge an expiration time for untreated CFEs has not been reported, and the present results should ease the concerns of researchers and save resources.

The present results also indicate that cleaning CFEs prior to use, at least with solvents such as isopropanol or xylene, is not necessary or beneficial to the CFE quality in regards to dopamine measurements. The sensitivity indexes generated from in vitro calibrations of CFEs pre-cleaned with either isopropanol or xylene were not significantly different than those of untreated CFEs (Figure 1(c)). The 
CFEs in the present study were stored uncovered in a room designated for electrode construction. As mentioned above, our CFEs were constructed such that the pulled-glass formed a tight seal around the carbon fiber, with no need for a sealant. Some researchers use an adhesive, such as epoxy or paraffin, to aide in forming this seal and, therefore, need a solvent to remove these substances and expose the carbon fiber tip [9] [13]. In fact, solvents such as 2-propanol and xylene have been shown to improve CFE responses during FSCV recordings [12] [13]; however, we were unable to replicate these findings using fixed potential amperometry.

The in vitro calibration process itself did not affect CFE sensitivity in the present study. In regards to dopamine measurements, CFEs from the in vitro exposure group had similar sensitivity indexes compared to untreated CFEs (Figure 1(c)). These findings support the practice of calibrating CFEs prior to experimental use, which requires increased preparation upfront but can save valuable time during experiments, especially important when animals are involved. Some manufacturers of commercially available CFEs pre-calibrate the electrodes in order to ensure quality, and the present findings suggest this likely does not reduce the CFE sensitivity for the customer. Further experiments are needed to clarify whether longer in vitro use affects CFE sensitivity.

Many researchers calibrate CFEs following in vivo experimental use [6] [11]. The present findings indicate CFE use during in vivo amperometric dopamine recordings in brain tissue decreases sensitivity compared to unused CFEs (Figure 1(c)). Logman et al. (2000) used in vivo microdialysis in combination with FSCV to determine whether pre- or post-experiment calibrations of carbon fiber microdisk electrodes give the most accurate representation of the actual concentrations measured in the brain [8]. Their findings suggest that pre-calibration results in a better estimate of the actual concentration of measured analyte, which in their project was acetaminophen. Furthermore, they conclude that the electrode is likely fouled during removal from the brain, the point at which blood and other fluids accumulate on the electrode and consequently lead to a lower post-calibration response [8]. The present results suggest that cleaning CFEs following in vivo experimental exposure negates this fouling effect and restores CFE sensitivity (Figure 1(c)). Both solvents tested, isopropanol and xylene, brought the sensitivity of the in vivo exposure group back to statistical similarity with the untreated CFEs. Considering the environmental and biological hazards associated with xylene [16], isopropanol is the safer solution to sensitivity issues in post-calibration.

\section{Conclusion}

In conclusion, in regards to amperometric measurements of dopamine efflux, the present findings confirm that CFEs maintain sensitivity for at least 2 months following construction, cleaning CFEs with isopropanol or xylene prior to use does not affect sensitivity, and in vitro calibration of CFEs does not alter sensi- 
tivity. In vivo experimental exposure in brain tissue did reduce CFE sensitivity, but this effect is negated if CFEs are cleaned with either isopropanol or xylene following use in brain tissue. In vitro calibrations of CFEs used with fixed potential amperometry or FSCV are valuable for normalizing CFE output in order to compare between animals/experiments. However, given the variability of these electrodes across labs and the handling factors that may alter sensitivity, caution should be used when trying to claim absolute neurotransmitter concentrations. The present experiments were only conducted using amperometry, not voltammetry; thus, the results may be limited in generalizability. FSCV has absorption issues related to the integrity of the carbon fiber surface that are not a factor in fixed potential amperometry. However, in an effort to standardize CFE use and explain discrepancies between researchers, the present study increased understanding of factors affecting CFE sensitivity.

\section{References}

[1] Gonon, F., Buda, M., Cespuglio, R., Jouvet, M. and Pujol, J.F. (1980) In Vivo Electrochemical Detection of Catechols in the Neostriatum of Anaesthetized Rats: Dopamine or DOPAC? Nature, 286, 902-904. https://doi.org/10.1038/286902a0

[2] Ponchon, J.L., Cespuglio, R., Gonon, F., Jouvet, M. and Pujol, J.F. (1979) Normal pulse Polarography with Carbon Fiber Electrodes for In Vitro and In Vivo Determination of Catecholamines. Analytical Chemistry, 51, 1483-1486.

https://doi.org/10.1021/ac50045a030

[3] McCreery, R.L. (2008) Advanced Carbon Electrode Materials for Molecular Electrochemistry. Chemical Reviews, 108, 2646-2687.

https://doi.org/10.1021/cr068076m

[4] Dayton, M.A., Ewing, A.G. and Wightman, R.M. (1981) Evaluation of Amphetamine-Induced In Vivo Electrochemical Response. European Journal of Pharmacology, 75, 141-144. https://doi.org/10.1016/0014-2999(81)90074-1

[5] Dugast, C., Suaud-Chagny, M.F. and Gonon, F. (1994) Continuous In Vivo Monitoring of Evoked Dopamine Release in the Rat Nucleus Accumbens by Amperometry. Neuroscience, 62, 647-654. https://doi.org/10.1016/0306-4522(94)90466-9

[6] Fielding, J.R., Rogers, T.D., Meyer, A.E., Miller, M.M., Nelms, J.L., Mittleman, G., Blaha, C.D. and Sable, H.J.K. (2013) Stimulation-Evoked Dopamine Release in the Nucleus Accumbens Following Cocaine Administration in Rats Perinatally Exposed to Polychlorinated Biphenyls. Toxicological Sciences, 136, 144-153. https://doi.org/10.1093/toxsci/kft171

[7] Lester, D.B., Rogers, T.D. and Blaha, C.D. (2010) Acetylcholine-Dopamine Interactions in the Pathophysiology and Treatment of CNS Disorders. CNS Neuroscience and Therapeutics, 16, 137-162. https://doi.org/10.1111/j.1755-5949.2010.00142.x

[8] Logman, M.J., Budygin, E.A., Gainetdinov, R.R. and Wightman, R.M. (2000) Quantitation of In Vivo Measurements with Carbon Fiber Microelectrodes. Journal of Neuroscience Methods, 95, 95-102. https://doi.org/10.1016/S0165-0270(99)00155-7

[9] Rodeberg, N.T., Sandberg, S.G., Johnson, J.A., Phillips, P.E.M. and Wightman, R.M. (2017) Hitchhiker's Guide to Voltammetry: Acute and Chronic Electrodes for In Vivo Fast-Scan Cyclic Voltammetry. ACS Chemical Neuroscience, 8, 221-234. https://doi.org/10.1021/acschemneuro.6b00393 
[10] Benoit-Marand, M., Suaud-Chagny, M.F. and Gonon, F. (2007) Presynaptic Regulation of Extracellular Ddopamine as Studied by Continuous Amperometry in Anesthetized Animals. In: Michael, A.C. and Borland, L.M., Eds., Electrochemical Methods for Neuroscience, CRC Press, Boca Raton, Chapter 3.

[11] Michael, D.J. and Wightman, R.M. (1999) Electrochemical Monitoring of Biogenic Amine Neurotransmission in Real Time. Journal of Pharmaceutical and Biomedical Analysis, 19, 33-46. https://doi.org/10.1016/S0731-7085(98)00145-9

[12] Bath, B.D., Michael, D.J., Trafton, B.J., Joseph, J.D., Runnels, P.L. and Wightman, R.M. (2000) Subsecond Adsorption and Desorption of Dopamine at Carbon-Fiber Microelectrodes. Analytical Chemistry, 72, 5994-6002. https://doi.org/10.1021/ac000849y

[13] Ramsson, E.S., Cholger, D., Dionise, A., Poirier, N., Andrus, A. and Curtiss, R. (2015) Characterization of Fast-Scan Cyclic Voltammetric Electrodes Using Paraffin as an Effective Sealant with In Vitro and In Vivo Applications. PLoS One, 10, e0141340. https://doi.org/10.1371/journal.pone.0141340

[14] Budai, D. (2010) Carbon Fiber-Based Microelectrodes and Microbiosensors. In Intelligent and Biosensors. InTech. https://doi.org/10.5772/7158

[15] Zhang, D.A., Rand, E., Marsh, M., Andrews, R.J., Lee, K.H., Meyyappan, M. and Koehne, J.E. (2013) Carbon Nanofiber Electrode for Neurochemical Monitoring. Molecular Neurobiology, 48, 380-385. https://doi.org/10.1007/s12035-013-8531-6

[16] Niaz, K., Bahadar, H., Magbool, F. and Abdollahi, M. (2015) A Review of Environmental and Occupational Exposure to Xylene and Its Health Concerns. EXCLI Journal, 14, 1167-1186. 